\title{
Response of Bowenoid Papulosis to Combination Treatment of Oral Isotretinoin and Topical 1\% 5-Fluorouracil
}

\author{
Shravya Rimmalapudi ${ }^{1}$, Sugat Jawade ${ }^{2}$, Bhushan Madke ${ }^{3}$, Adarsh Lata Singh ${ }^{4}$ \\ 1, 2, 3, 4 Department of Dermatology, Venereology and Leprosy, Jawaharlal Nehru Medical College, \\ Datta Meghe Institute of Medical Science, Wardha, Maharashtra, India.
}

\section{INTRODUCTION}

Bowenoid papulosis (BP) is a rare benign disease which can have spontaneous regression but can sometimes turn malignant. ${ }^{1}$ It is characterised by solitary or multiple verruca-like papules or plaques usually present on genitalia having a close histological resemblance to Bowen's disease and a predilection for sexually active young adults. ${ }^{2}$ It affects all races equally and has almost same male to female ratio. There are an estimated 5 cases per 100000 women. The exact prevalence is unknown. ${ }^{3}$ We hereby present a case report of bowenoid papulosis.

Bowenoid papulosis is an uncommon form of intraepithelial neoplasia. It is caused by human papilloma virus (HPV) infection and clinically resembles viral wart while histologically resembling in situ squamous cell carcinoma (SCC). We hereby report a case of an adult male with multiple verrucous lesions on penis and scrotum. The histopathology showed features of bowenoid papulosis. The patient was treated with oral isotretinoin and topical $1 \% 5$ - fluorouracil following which the lesions reduced remarkably in the span of 3 weeks.

\section{PRESENTATION OF CASE}

A 48-year-old immunocompetent male came to our out-patient department (OPD) with a 6 months history of multiple fleshy lesions over penis and scrotum associated with itching which was mild in nature and intermittent. Initially there was a single pea sized lesion on the shaft of penis which was insidious in onset and gradually progressed in size and number onto base of penis and scrotum over a period of two months. Earlier treatment with topical steroids showed no improvement. There is no involvement of oral mucosa. There was no family history of skin cancers or similar history in partner. Patient doesn't report any high-risk sexual behaviour or history of smoking.

Cutaneous examination showed multiple, well defined, verrucous fleshy papules and plaques with hyperpigmented borders over shaft of penis, base of penis and scrotum of size $4 \times 2 \mathrm{~cm}, 1 \times 0.5 \mathrm{~cm}, 2 \times 2 \mathrm{~cm}$ respectively (Figure1,). Complete blood count (CBC) and random blood sugar (RBS) were within normal limits. Blood serology tests including venereal disease research laboratory test were non-reactive. A $4 \mathrm{~mm}$ skin biopsy revealed parakeratosis, moderate epidermal hyperplasia along with dysplasia, irregular acanthosis, papillomatosis and koilocytosis (Figure2).

The lesions were treated with oral isotretinoin $20 \mathrm{mg}$ once daily and topical $1 \%$ 5 - fluorouracil cream twice daily. After 3 weeks of treatment the lesions became flattened and reduced in size (Figure3). The patient and his partner were advised for a regular follow up.
Corresponding Author: Dr. Rimmalapudi Shravya, Dermatology, Venereology \& Leprosy, AVBRH, Datta Meghe Institute of Medical Sciences, Sawangi (Meghe), Wardha, Maharashtra, India. E-mail: rsshrav3@gmail.com

DOI: $10.14260 / j e m d s / 2021 / 625$

How to Cite This Article:

Rimmalapudi S, Jawade S, Madke B, et al. Response of bowenoid papulosis to combination treatment of oral isotretinoin and topical 1\% 5 - fluorouracil. J Evolution Med Dent Sci 2021;10(35):3067-3069, DOI: 10.14260/jemds/2021/625

Submission 09-09-2020,

Peer Review 24-07-2021

Acceptance 30-07-2021,

Published 30-08-2021.

Copyright (C) 2021 Shravya Rimmalapudi et al. This is an open access article distributed under Creative Commons Attribution License [Attribution 4.0 International (CC BY 4.0)] 

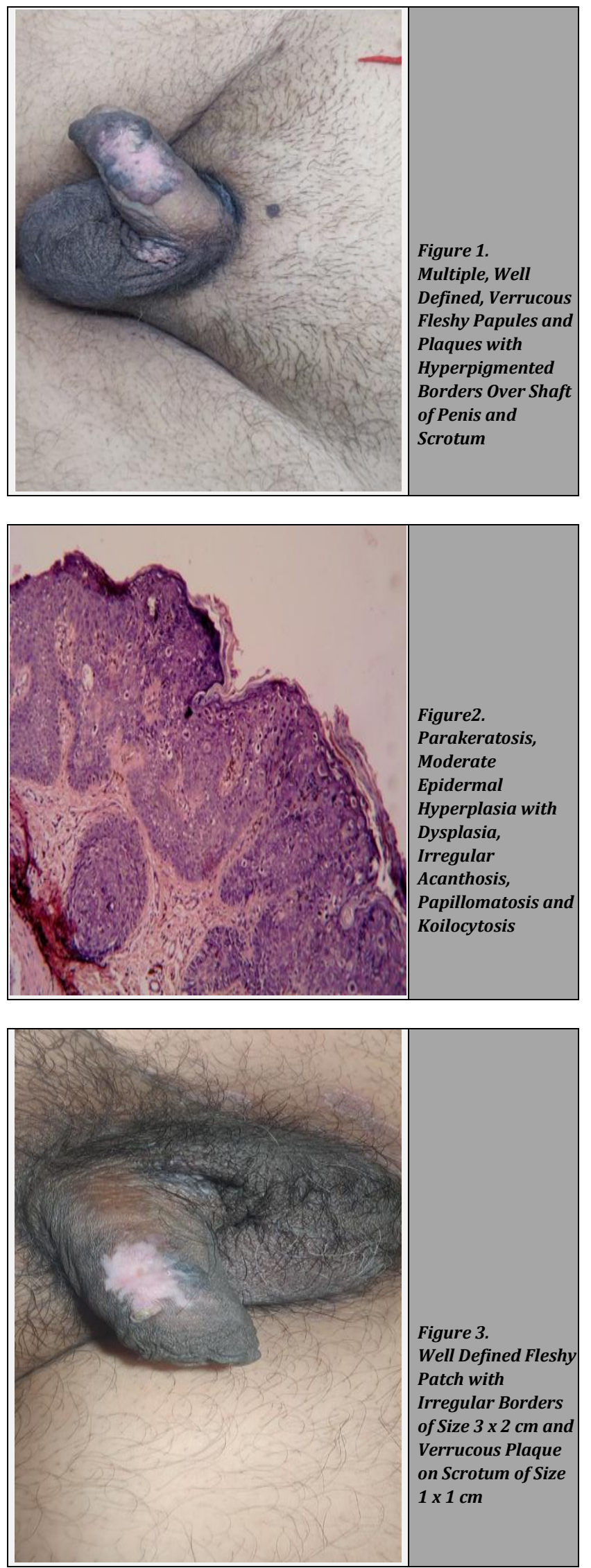

\section{DISCUSSION}

Bowenoid papulosis is an uncommon sexually transmitted condition which is a focal epidermal hyperplasia and dysplasia associated with human papilloma virus. ${ }^{3}$ It is a premalignant lesion and was first described by Lloyd but described as an entity by Kopf et al. ${ }^{1}$ It is an uncommon form of intraepithelial neoplasia. The term "intraepithelial neoplasia" defines an intraepithelial change within anogenital epithelium that is premalignant. Immune response appears to play a role in the development of BP.

It is common in sexually active adults mostly in their third to the mid-fifth decade with a mean age of 31 years with slight female predominance. ${ }^{4,5}$ Smoking has been known as a recurrence factor. The exact prevalence is unknown because the lesions are clinically related to genital warts. ${ }^{3}$

Bowenoid papulosis is caused by human papilloma virus of serotypes $18,31,33,39$ and 52 of which 16,18 , and 33 are the most oncogenic strains. It must be distinguished from inflammatory conditions such as lichen planus, seborrhoeic keratosis, melanocytic nevi, condyloma acuminatum, Bowen disease, warty dyskeratoma, zoon balanitis or vulvitis, and cutaneous malignancies like invasive SCC. ${ }^{2}$

Clinically it presents as multiple well-demarcated skin coloured to violaceous papules, usually less than $1 \mathrm{~cm}$ in size commonly over genitalia. The most common sites involved are the vulva and penis but sometimes other sites like periungual, oeal mucosa, lower abdomen and neck may be involved. ${ }^{1}$ The surface of the lesion can be smooth, plane topped, verrucous or papillomatous. Some papules may coalesce into plaques. Sometimes it presents as warty white plaques.,4 Lesions are usually asymptomatic but can itch occasionally. Bowenoid papulosis is a premalignant lesion and is considered as a transitional stage between squamous carcinoma in situ and genital warts.

Clinical variants of BP such as papules coalescing to form plaques, lichenoid papules, macules, and leukoplakia-like lesions have been described. ${ }^{1}$ Bowenoid papulosis, erythroplasia of Queyrat and Bowen's disease are distinct clinical entities with similar histopathological findings of intraepithelial neoplasia, but with different clinical behaviour and risks of progression to invasive squamous cell carcinoma hence it's necessary to differentiate the two.

Histopathology of BP is characterized by epidermal hyperplasia and dysplasia, hyperkeratosis with parakeratosis and acanthosis with partial epidermal atypia (characterised by circumscribed epidermal proliferation composed of pleomorphic cells with clumped and irregular nuclei) and spongiosis. The integrity of the dermo epidermal border is preserved.

The pattern occasionally has been described as windblown and maybe identical to Bowen disease. Dermis shows tortuous and dilated capillaries with lymphohistiocytic infiltrate. Features such as extent of epidermal atypia and characteristics of koilocytes are important in the diagnosis of Bowenoid papulosis. ${ }^{6}$ Kaziouskaya et al. studied the expression of p16 protein in bowenoid papulosis which is concluded in having high sensitivity and specificity. HPV subtyping is not performed routinely in bowenoid papulosis.

The course of this disease ranges from spontaneous regression to rare progression to invasive squamous cell carcinoma. The lesions can regress in few months, persist for years, recur or progress to invasive SCC, especially in immunocompromised individuals. ${ }^{2}$ Younger patients tend to have a self-limiting course lasting months. 
There are different treatment options in this condition which can be through local route such as application of imiquimod, podophyllin, 5 fluorouracil and cidofovir. ${ }^{4}$ Surgical modalities include shave biopsy, electrodessication, cryotherapy, surgical excision, interferon injection and vaporization by laser. ${ }^{2}$

Retinoid either topically or systemically is also effective in treating this condition. Immunomodulators like imiquimod 5 $\%$, interferon beta may lengthen the remission period of lesions and may decrease the relapse rate. Therapeutic vaccination has also been tried.

There have been few studies on bowenoid papulosis of genitalia. In one of the studies, the treatment included only topical application of $1 \% 5$ - fluorouracil and there was excellent response at end of 2 weeks. ${ }^{2}$ Another study reported two cases which were treated with topical tazarotene $0.05 \%$ gel and both of them achieved clearing of lesions but there was recurrence in one. 4

Our patient had bowenoid papulosis which was confirmed clinically and histologically. The patient was treated with topical $1 \% 5$ - fluorouracil and oral isotretinoin once daily. The lesions reduced remarkably after the treatment in the span of 3 weeks with only side effect being dryness. No recurrence was observed during the follow up of 3 months.

5 - fluorouracil is a fluorinated pyrimidine analog with cytotoxic effects used in multiple AKs and superficial basal cell carcinoma (BCCs). Isotretinoin is a first generation nonaromatic retinoids and affects pathways involved in inflammation, cellular differentiation, apoptosis, and sebaceous gland activity. It is mainly indicated in acne, psoriasis etc.

Studies are available on different kind of skin lesions, dermatophytoses ${ }^{7-9}$ and related drug trials. ${ }^{10-12}$ Deshmukh et al. studied about treatment of genital warts with a combination of cryotherapy and $5 \%$ imiquimod. ${ }^{12}$ Studies and cases on pemphigus, $^{13}$ psoriasis $^{14}$ and reactive arthritis ${ }^{15}$ have been reported.

\section{CONCLUSIONS}

Bowenoid papulosis is a benign condition with good prognosis in majority of the patients, it's possible malignant potential and frequency rate of recurrence should not be disregarded. Hence, early diagnosis followed by adequate treatment is necessary. The patient should be thoroughly educated regarding HPV infection, the risk of its malignant potential and the use of condoms. They should be frequently examined for recurrence and a follow up treatment is necessary every 3 - 6 months if the lesions recur or do not resolve. In conclusion, this case emphasizes on insidious onset of BP lesions, the possible evolution into malignancy, a successful medical therapy achieving remarkable reduction in lesions and the importance of its awareness among sexually active individuals.

Financial or other competing interests: None.

Disclosure forms provided by the authors are available with the full text of this article at jemds.com.

\section{REFERENCES}

[1] Nayak SUK, Shenoi SD, Bhat ST, et al. Bowenoid papulosis. Indian J Sex Transm Dis AIDS 2015;36(2):2235.

[2] Shivanna R, Kapoor M, Murthy BN, et al. Bowenoid papulosis of genitalia responding to topical 5Fluorouracil. Clin Dermatol Rev 2018;2(1):25-7.

[3] Chamli A, Zaouak A. Bowenoid papulosis. In: StatPearls. Treasure Island (FL): StatPearls Publishing 2021 Jan. [Last update Jul 18, 2021].

[4] Shastry V, Betkerur J, Kushalappa. Bowenoid papulosis of the genitalia successfully treated with topical tazarotene: a report of two cases. Indian J Dermatol 2009;54(3):2836.

[5] Gaidhane AM, Sinha A, Khatib MN, et al. A systematic review on effect of electronic media on diet, exercise and sexual activity among adolescents. Indian Journal of Community Medicine 2018;43(Suppl 1):S56-65.

[6] Gross G, Ikenberg H, Gissmann L, et al. Papillomavirus infection of the anogenital region: correlation between histology, clinical picture and virus type. Proposal of a new nomenclature. J Invest Dermatol 1985;85(2):147-52.

[7] Laad G, Madke B, Nikam B. Cirsoid aneurysm of upper eyelid. Journal of Dermatology and Dermatologic Surgery 2019;23(2):109-10.

[8] Kedia P, Madke B. Unilateral molluscum contagiosum following eyebrow grooming. Journal of Clinical and Diagnostic Research 2019;13(11):WD01-2.

[9] Begum J, Mir NA, Lingaraju MC, et al. Recent advances in the diagnosis of dermatophytosis. Journal of Basic Microbiology 2020;60(4):293-303.

[10] Sahu PJ, Singh AL, Kulkarni S, et al. Study of oral tranexamic acid, topical tranexamic acid and modified Kligman's regimen in treatment of Melasma. Journal of Cosmetic Dermatology 2020;19(6):1456-62.

[11] Varsha G, Yeshwant L. Minoxidil a youth elixir for eyebrow hypotrichosis. Journal of Clinical and Diagnostic Research 2020;14(2):PC01-3.

[12] Deshmukh S, Sudhir S, Sugat J, et al. Treatment of genital warts with a combination of cryotherapy and 5\% imiquimod. Journal of Clinical and Diagnostic Research 2020;14(2):WD01-3.

[13] Sandeep K, Pooja JS, Aishwarya DP, et al. Neonatal pemphigus in a neonate of a mother suffering from pemphigus vulgaris- a case presentation. Journal of Clinical and Diagnostic Research 2020;14(1):WD01-3.

[14] Kute PK, Muddeshwar MG, Sonare AR. Pro-oxidant and anti-oxidant status in patients of psoriasis with relation to smoking and alcoholism. Journal of Evolution of Medical and Dental Sciences 2019;8(34):2677-80.

[15] Amruta DM, Bhushan SM, Adarsh LS, et al. Response to infliximab biosimilar in a case of reactive arthritis: our experience. Journal of Clinical and Diagnostic Research 2019;13(12):WL01-2. 